\title{
Introduction
}

\section{Conception de documents numériques : parcours méthodologique}

\author{
Stéphane Caro Dambreville
}

Département Services et Réseaux de Communication

IUT de Dijon - Université de Bourgogne

Laboratoire CIMEOS EA 4177 équipe LIMSIC

Bd Dr. Petitjean

F-21078 Dijon cedex

stephane.caro@u-bourgogne.fr

http://lescamot-scd.blogspot.com/

\begin{abstract}
RÉSUMÉ. Les travaux de recherche relatifs à la conception de documents numériques répondent à une forte demande sociale. Pourtant nos modèles de société et éducatifs ont du mal à intégrer les enjeux liés à ces types de documents. Cela étant, l'objet est jeune, moins d'un demi siècle, il n'y a donc rien d'exceptionnel à ce que les pratiques d'écriture et de formation soient seulement en cours d'élaboration. Comme le livre, le document numérique est un type de document qui peut mobiliser de nombreux métiers pour son élaboration. La palette de ces métiers tend à s'accroitre constamment : ergonomes, référenceurs, architectes de l'information, programmeurs, graphistes, intégrateurs... Il est par conséquent difficile d'avoir une vision globale sur tous ces savoir-faire et les méthodologies afférentes. Ce numéro spécial n'a d'autre prétention que de présenter une partie de ces méthodes propres à la conception de documents numériques. Ce faisant il contribue à rendre visible des techniques qui sont employées par différents spécialistes du domaine dans le cadre de leur activité. Nous ne traiterons ici que certaines phases qui précèdent la fabrication à l'aide d'outils logiciels, de langages de programmation ou de description de page.
\end{abstract}

ABSTRACT. There is a strong social demand for studies of digital document. Yet our models of society and education are struggling to integrate issues related to these types of documents. However, the subject is less than half a century old, and so it is hardly surprising that the writing and training practices are just being developed. Like the book, the digital document involves many different skills and techniques in its development. The list of jobs tends to grow steadily: ergonomists, referencing specialists, information architects, programmers, designers, integrators ... It is therefore difficult to have a global view on all this know-how and the related methodologies. This special edition seeks to present some of these methods involved in digital documents design. In doing so it helps to make visible the techniques that 
2 Document numérique. Volume $12-\mathrm{n}^{\circ} 2 / 2009$

are employed by various experts in the field as part of their business. We deal here only with some pre-production phases using software tools, programming languages or page description.

MOTS-CLÉS : Méthodes de conception de documents numériques, design de documents numériques, architecture de l'information, scénarisation de documents numériques, organisateurs de navigation, organisateurs de structure, organisateurs de mise en relief de l'information, sélection de l'information.

KEYWORDS: Design methods of digital documents, digital document design, information architecture, storyboarding digital documents, navigation organizers, structural organizers, information hierarchycal organizers, information selection. 


\section{Public cible}

Ce numéro spécial s'adresse aux acteurs de la conception de documents numériques, qu'ils soient professionnels, chercheurs, enseignants. Pour simplifier le propos nous désigneront par « l'agence » la cible principale de cet article introductif. La visée n'est pas exhaustive, même si, pour cette introduction, nous nous efforcerons de donner une vision d'ensemble de diverses méthodologies utilisées en matière de conception de documents numériques. Les articles présentés ensuite en illustreront certains aspects de manière plus approfondie. Le point de vue adopté consiste à formaliser une démarche globale de conception qui ne soit pas trop lourde et qui à certains moment clés mobilise des outils qui permettent de prendre en compte certain(e)s besoins/préférences des utilisateurs mais aussi le respect des standards existants. Certaines démarches d'ingénierie cognitive, d'analyse et de conception globales (Poulson et al., 1996) sont difficiles à appréhender dans la réalité de nombreux projets de petite et moyenne envergure. Nous présentons ici une démarche qui place le concepteur face à ses besoins d'analyse tout en intégrant en parallèle des dimensions propres à l'usager. Bien entendu pour des projets conséquents en taille et en enjeux (sécurité, fiabilité) des démarches plus formelles intégrant d'autres méthodes de conception pourront être mobilisées. Toutefois dans ces cas de figure, il faudra bien souvent faire appel à des spécialistes de l'ingénierie cognitive.

\section{Le processus de conception}

Le processus de conception peut s'envisager pour le concepteur du plus général au particulier comme sur la figure 1 . 


\section{Phases $\quad$ Livrables}

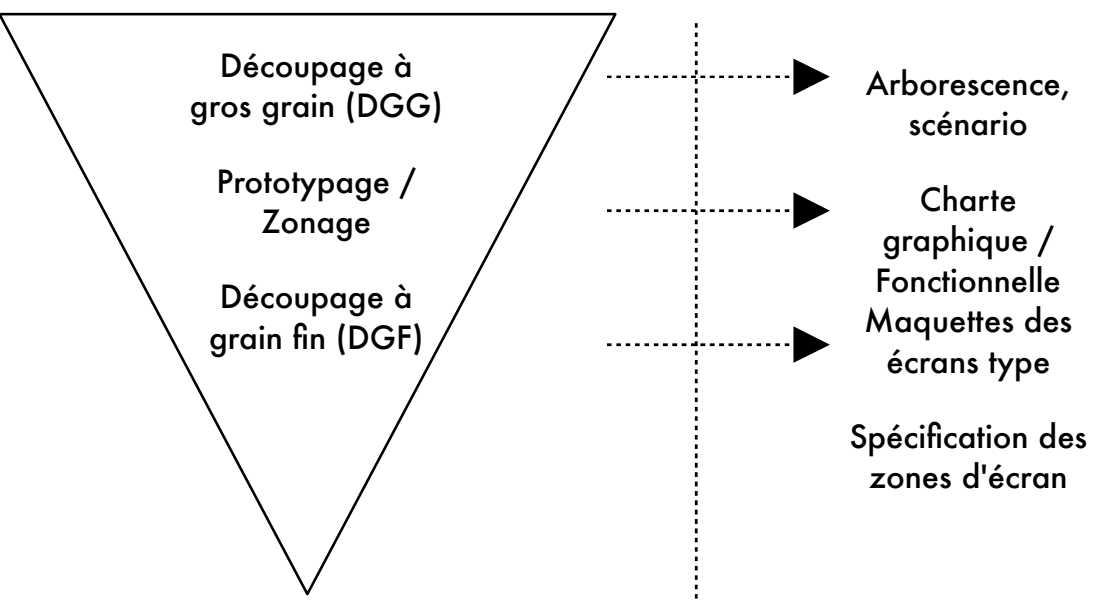

Figure 1. Cycle de conception en $V$

La première phase va servir à organiser l'information sous la forme d'une structure de navigation. Cette structure donnera lieu à une arborescence, un schéma de navigation ou d'autres formes de représentation de l'information plus moléculaires dans le cas de scénarios par exemple. Cette macro structuration n'est pas visible mais perceptible lors des processus de navigation qui organiseront l'accès à l'information à l'aide d'organisateurs de structure et de navigation. Cette première phase a deux objectifs, la segmentation de l'information et sa représentation formelle, puis l'identification d'un certains nombres d'espaces (ou de pages par analogie au livre) ayant des caractéristiques communes. Ces pages ou « écrans types » donneront lieu à un prototypage spécifique (page d'accueil, plan du site, formulaire, page produit etc.) qui constituera une seconde phase. A un niveau inférieur on aura le souci, pour chaque type d'écran, d'organiser l'information et de la mettre en forme à l'aide d'organisateurs de mise en relief de l'information (mise en avant $v s$ mise en retrait). On sera particulièrement attentif aux zones de chaque écran type et en particulier à la zone d'information principale des pages liées à la présentation $\mathrm{du}$ « contenu » proprement dit. 


\section{Etape 1 Découpage à gros grain ${ }^{1}$ (DGG) : macro-découpage de l'information}

\subsection{Procédure}

La question du découpage de l'information pour la mise en écran n'est pas nouvelle. Les sumériens qui écrivaient sur des tablettes d'argile il y a 4000 ans se posaient vraisemblablement les mêmes questions que nous. Comment disposer des informations dans un espace limité ? Le découpage à gros grain (DGG désormais) de l'information consiste à considérer (approximativement) l'ensemble des informations ou des types de contenus à présenter dans le document et à les organiser de manière à en permettre l'accès. Trois cas de figure peuvent se présenter :

- Le DGG est imposé / proposé par le commanditaire (sous forme d'arborescence ou de liste).

- Le DGG est contraint par un scénario (ludique, pédagogique).

- Le DGG est à la charge de l'agence.

Dans tous les cas et surtout quand le découpage est à la charge de l'agence, on veillera dans une phase préalable à identifier les types d'utilisateurs, la tâche et le contexte d'utilisation dont va faire l'objet le document. Ces facteurs pourront être présentés dès le cahier des charges et permettront de statuer sur la pertinence du DGG.

Par exemple, dans le cas de la réalisation d'un document de formation à la recherche d'information commandé par le service commun de documentation d'une université, on pourra brièvement décrire ces éléments de la manière suivante :

- Utilisateurs : étudiants de niveau (Licence) L1, L2, L3

- Tâche : Maîtriser la recherche d'information sur internet

- Contexte : Utilisation du cours en autonomie à l'université ou en dehors. Utilisation du cours comme support de formation pour des activités encadrées en groupes avec un formateur

\subsection{DGG fourni par le commanditaire}

Le macro découpage, quand il est fourni par le commanditaire peut toutefois être perfectible. Des rubriques et sous rubriques peuvent avoir le même nom par

${ }^{1}$ Le découpage à gros grain ou à grain fin peuvent être remplacés par macro découpage ou micro découpage. Il ne semble pas y avoir de consensus sur l'expression à employer pour désigner ces concepts. La référence à une macro-structure du document peut être ambiguë du fait de l'emploi du même concept en psychologie de la compréhension dans le modèle de lecture / compréhension de Kintsch (Kintsch et al., 1978), (Kintsch, 1988, 1998). De ce fait, nous utiliserons la variation du grain, expression qui ne semble pas employée dans la littérature pour ce type de concept mais qui convient à notre propos. 
exemple. L'organisation peut ne pas être adaptée aux utilisateurs ciblés (organisation, vocabulaire) où à la tâche, au contexte. Dans ce cas, le rôle de l'agence pourra être de valider ce DGG et au besoin attirer l'attention du commanditaire sur les éventuelles faiblesse du découpage fourni. Dans cette perspective, on pourra utiliser la méthode du tri de cartes pour valider ou non l'arborescence proposée. On consultera valablement, dans ce numéro spécial, l'article de Fastrez P. Campion B. et Collard A.-S. qui présente la méthode du tri de cartes appliquée à la définition de l'architecture du site web d'un département universitaire.

\subsection{DGG contraint par un scénario}

Le découpage à gros grain peut être dépendant d'une séquence scénarisée et répondre à d'autres logiques que celles qui peuvent être inhérentes à une structure de navigation traditionnelle dépendante des liens prévus. Dans cette perspective il convient d'adapter la répartition du contenu dans des séquences qui peuvent avoir une présentation formelle moléculaire ou atomique et répondre à des fonctionnements «programmés ». L'article de Durand A. part de ce postulat et l'applique à la formalisation moléculaire pour l'écriture de scenarii de documents multimédia.

\subsection{DGG conçu par l'agence}

Le découpage à gros grain peut faire partie de la commande et être proposé par l'agence. Dans ce cas, l'étude préalable au projet devra intégrer cet aspect et selon l'objet du document (procédural : ex vente en ligne, procédure de SAV, ou connaissance ex : site vitrine) on recourra à diverses méthodes de conception. Concernant les documents numériques à vocation pédagogique, une représentation des concepts à intégrer par les apprenants sous forme d'unités sémantiques ou d'ontologies pourra être mobilisée. (Cf. l'article de Aqqal A. Elkamoun N. Rensing C. Berraissoul A. Steinmetz R. dans ce numéro spécial sur la représentation formelle de contenus à vocation pédagogique). L'identification des utilisateurs ciblés et de leur besoins sera une étape importante pour l'obtention d'un DGG pertinent. On pourra recourir à la méthode des personas (simulation de profils d'utilisateurs fictifs, de leurs profils et besoins afférents) des staffs d'experts de communautés de pratique. Brangier E. Dinet J et Bastien J.-M. présentent dans leur article les théories participant à cette approche ainsi qu'un exemple d'application à la reconception d'une bibliothèque numérique sur la connaissance de la construction européenne. La figure 2 présente l'analyse préalable que doit mener l'agence dans le cas ou le DGG est à sa charge et quand cette analyse n'est pas fournie par le commanditaire. 


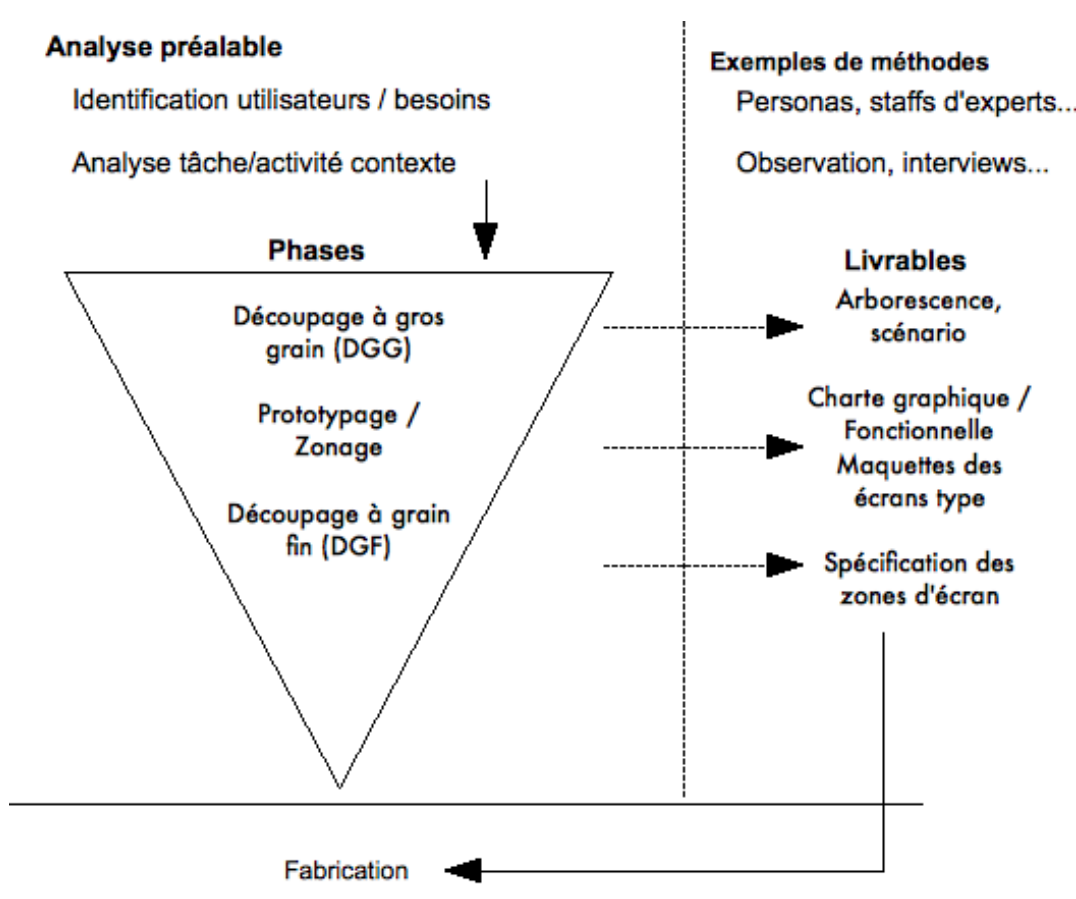

Figure 2. Analyse préalable / Cycle de conception / Fabrication 
Avec des documents ayant une vocation procédurale (exécution d'une procédure ou assistance à l'exécution : assistance à une opération de Service Après Vente par exemple), on utilisera des méthodes d'analyse de la tâche ou de l'activité. Les deux directions sont en général l'observation quand c'est possible et les interviews, entretiens, questionnaires. Concernant l'observation de la tâche à formaliser : on mobilisera les techniques liées à ce type de protocole : méthodologie de la psychologie expérimentale, verbalisation concomitante (Ericsson et al., 1993) (ou TPV technique des protocoles verbaux (Bisseret et al., 1999)), etc. Quand l'observation n'est pas possible ou souhaitable, on utilisera des méthodes d'interview ou de description formelle d'après un corpus textuel (entretiens, questionnaires) : TPC Technique du Pourquoi Comment (Bisseret et al., 1999), Méthodes d'analyse de tâches MAD (Modèle analytique de description des tâches utilisateur (Scapin et al., 1990)). La méthode MAD est un formalisme qui permet de décrire une procédure sous une forme proche d'un algorithme (état initial, condition, action etc. Pour les documents non procéduraux on pourra recourir à la méthode de tri de cartes évoquée plus haut, mais aussi à des formes de brain storming (TEA technique d'élicitation associative (Bisseret et al., 1999) ou des techniques de formalisation de schéma de catégorisation comme la technique des questions par alternative inspirée du jeu des 20 questions (TQA (Bisseret et al., 1999)).

\subsection{Livrables DGG : l'arborescence, le scénario}

Le macro découpage peut être formalisé par une arborescence sous forme d'organigramme, une liste sous forme d'énumération à retraits successifs, un réseau de noeuds, d'atomes et molécules etc. La figure 3 présente un exemple d'arborescence qui comporte aussi les types d'informations présentes sur les pages. 


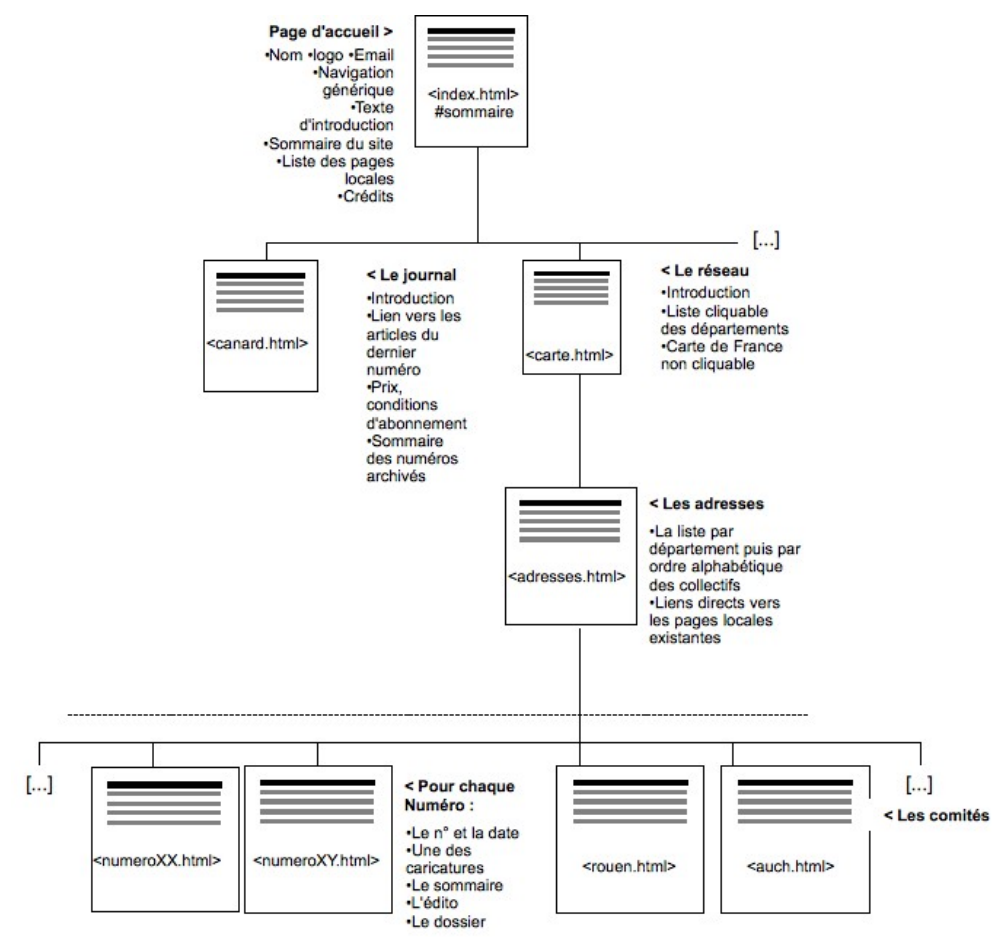

Figure 3. Arborescence. D'après «Conception, suivi et animation d'un site web associatif »- Eve Demazière - 1999 http://www.internatif.org

\subsection{Rendre utilisable le DGG}

\subsubsection{Les organisateurs de structure}

Les organisateurs de structure vont permettre l'accès à l'information et le repérage dans l'ensemble. Une image pertinente utilisée par Ronez est celle d'une commode aux tiroirs transparents dans laquelle on va ranger des vêtements (Ronez, 2007). Un document numérique professionnel doit avoir ce type de fonctionnement, c'est à dire un accès direct à l'information sans s'engager dans une démarche d'essai erreur (j'ouvre un tiroir et je le referme car ce n'est pas le bon).

La page web doit faire l'effet d'une commode aux tiroirs transparents. Pas question d'ouvrir successivement tous les tiroirs pour découvrir où se trouvent les chaussettes (Ronez, 2007).

Les organisateurs de structure ont aussi pour vocation de re-constituer artificiellement des dimensions «perdues » dans les documents numériques. Ces 
dimensions étaient souvent construites grâce à la possibilité de manipuler directement les documents papier (aspect tactile). L'indication du volume global du document, de l'endroit où l'on se trouve dans l'ensemble, le contexte (ce qui précède, ce qui suit), le volume de la partie courante etc.

Les organisateurs de structure facilitent la représentation mentale du document en vue d'éviter «surcharge cognitive» et « désorientation». Ces organisateurs « artificiels» peuvent être utilisés conjointement. Les organisateurs de structure peuvent être composés de dispositifs langagiers (par exemple « écran 1 sur 5 ») ou non langagiers (icônes, couleurs, aplats etc). Nous listons ici quelques exemples d'organisateurs d'aide à la construction d'un modèle mental de la structure du document que l'on peut utiliser pour améliorer l'utilisation de documents numériques :

- Les plans (cartes de site, cartes de navigation),

- Les indicateurs de positionnement (textuels : « écran 2 sur 12 » par ex.) ou sous forme graphique comme dans l'exemple ci-dessous (site Widil.com).

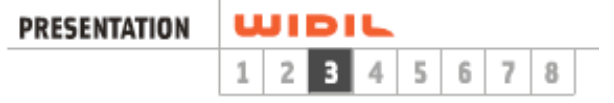

- Les indicateurs de volume (graphiques ou textuels : « ce chapitre comporte 15 écrans »),

- Les indicateurs de contexte (petit résumé textuel ou graphique qui permet de comprendre l'écran de façon autonome sans consulter les autres écrans)- Plans de sites

\subsubsection{Les organisateurs de navigation}

Les organisateurs de navigation facilitent l'orientation et les déplacements dans le document. Ces organisateurs permettent par exemple de connaître le chemin parcouru, de revenir à un point de référence.

Tous ces organisateurs «artificiels » peuvent être utilisés conjointement. Les organisateurs de navigation peuvent être composés d'éléments langagiers (Fil d'Ariane par exemple). Nous listons ici quelques exemples d'organisateurs d'aide à la navigation que l'on peut utiliser pour améliorer l'utilisation d'un document numérique.

- Historiques de la navigation (Les «miettes » ou fil d'Ariane par exemple $=>$ Université de Bourgogne $>$ Les formations $>$ Les masters $>$ Master 1 MASCI)

- Retour à un « Point de repère » (Page d'accueil, Plan du site)

- Dispositifs de progression séquentielle (boutons « tourne page », liens Suivant Précédent, par ex.) 
- Dispositifs de retour en arrière («retour», fonction «Précédent» des navigateurs ou lien Retour)

- Annonces de destination (site corlobe.tk)

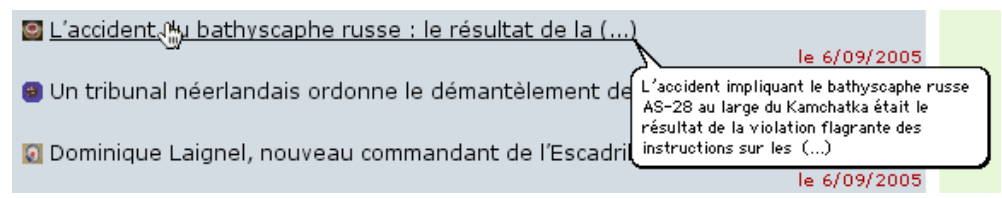

- Dispositifs d'information locale (escamots et infobulles par exemple).

- Dispositifs destinés à éviter les digressions, comme le regroupement des liens externes en bas d'écran.

- Filtres vue (site Crédit Mutuel)

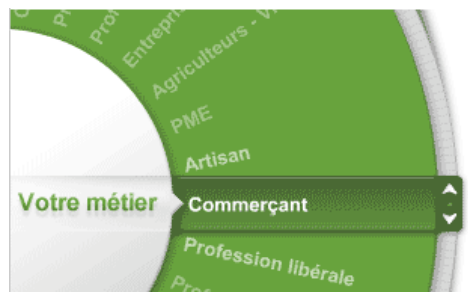

On peut aussi considérer les moteurs de recherche insérés à l'intérieur de sites Web dans cette optique, bien que ce ne soient pas à proprement parler des organisateurs, ils assurent une fonction analogue, assister la recherche et le parcours de l'information.

\subsubsection{Les organisateurs de structure et de navigation}

Le cloisonnement n'est pas tout à fait étanche entre les catégories d'organisateurs de structure et de navigation. D'une part, les organisateurs de structure quand ils remplissent bien leur rôle ont aussi une influence sur la navigation. D'autre part, certains de ces organisateurs ont une double ou triple fonction (indication du volume du document et de la localisation de l'endroit courant : par exemple «écran 1 sur $5 »)$. En voici 2 exemples sur la figure 4.

Indicateur de volume, de positionnement et dispositif de progression séquentielle 
12 Document numérique. Volume $12-\mathrm{n}^{\circ} 2 / 2009$

\section{Précédent Page $13 / 14 \varangle$ Suivant *

Précédent 1 | 2 | 3 || 4 || 5 | 6 | 7 | 8 | Suivant

Figure 4. Organisateurs de structure et de navigation sites Alain Guillemaud, La Redoute

\section{Etape 2 Prototypage / Zonage}

\subsection{Procédure}

Le prototypage / zonage consiste à maquetter les différents « écrans type » et à y placer les diverses zones d'information (On consultera l'article de Baccino T. dans ce numéro spécial sur le prototypage en ergonomie cognitive). Cet article présente cette démarche et le degré de fidélités des formes de prototypage. La figure 5 ci-dessous présente un prototype d'écran fourni par un commanditaire (Ecole Atelier des arts de la reliure «Les ateliers d'or») et la réalisation de la page d'accueil par l'agence (figure 6). 


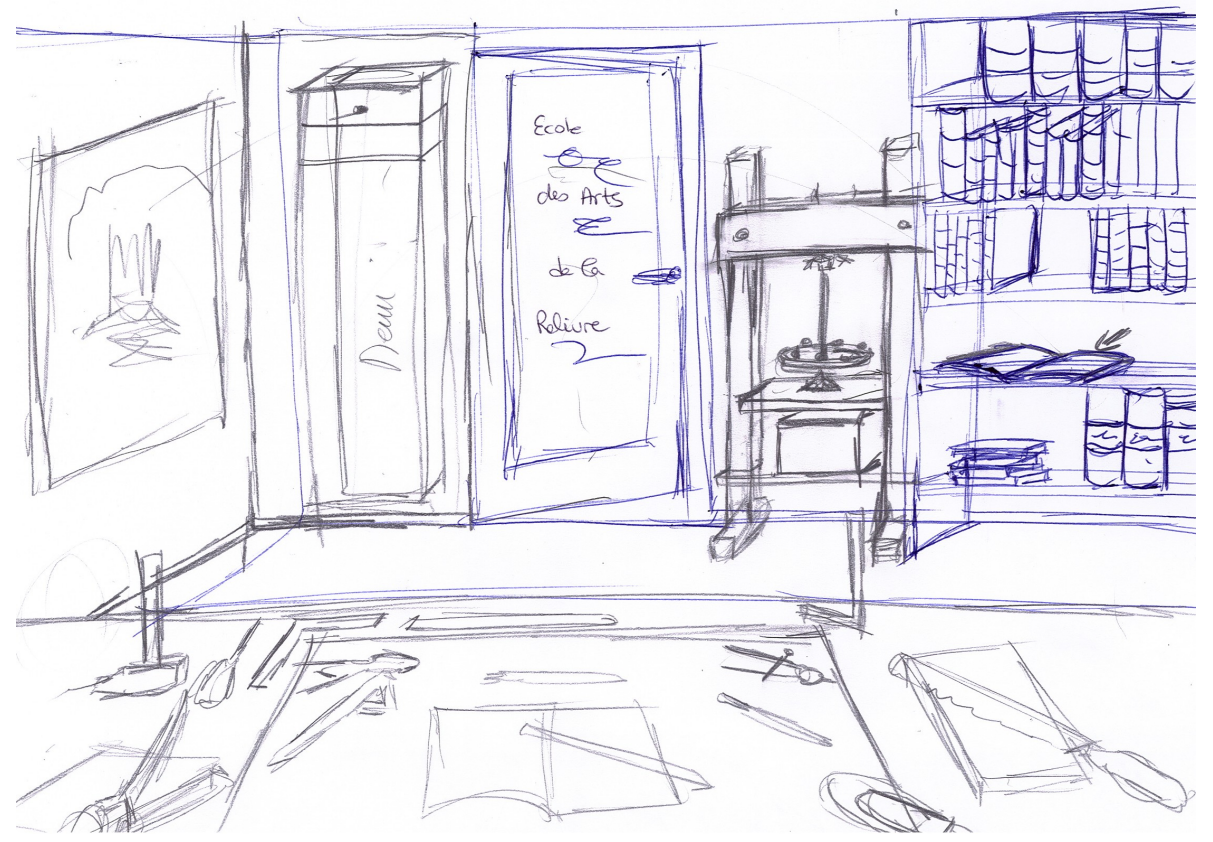

Figure 5. Prototypage/Zonage, maquette de la page d'accueil du site Ateliers d'or (E. Birlouez) 


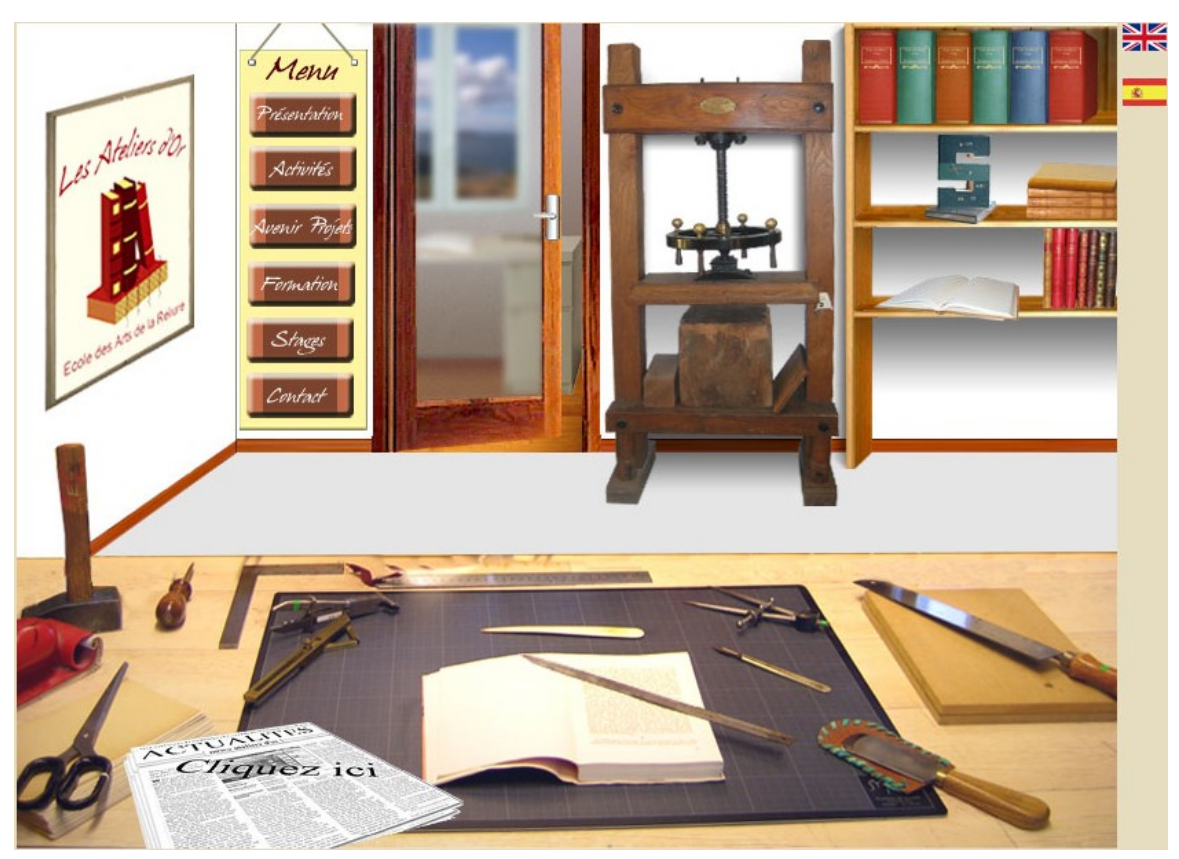

Figure 6. Prototypage / Zonage, page d'accueil du site Ateliers d'or (De Oliveira O. Daguenet A. Athimari M. Dufour L. Landau B.)

Les zones d'information peuvent donner lieu aussi à des modèles par type d'objet (tableaux, boutons, formulaires...). A ces maquettes fonctionnelles peuvent s'ajouter une recherche d'ambiance graphique en adéquation avec la demande du commanditaire. Cette recherche peut se faire à l'aide d'un concept board (association de textures, formes, objets, couleurs, typographies). La figure 7 présente un exemple de concept board. 


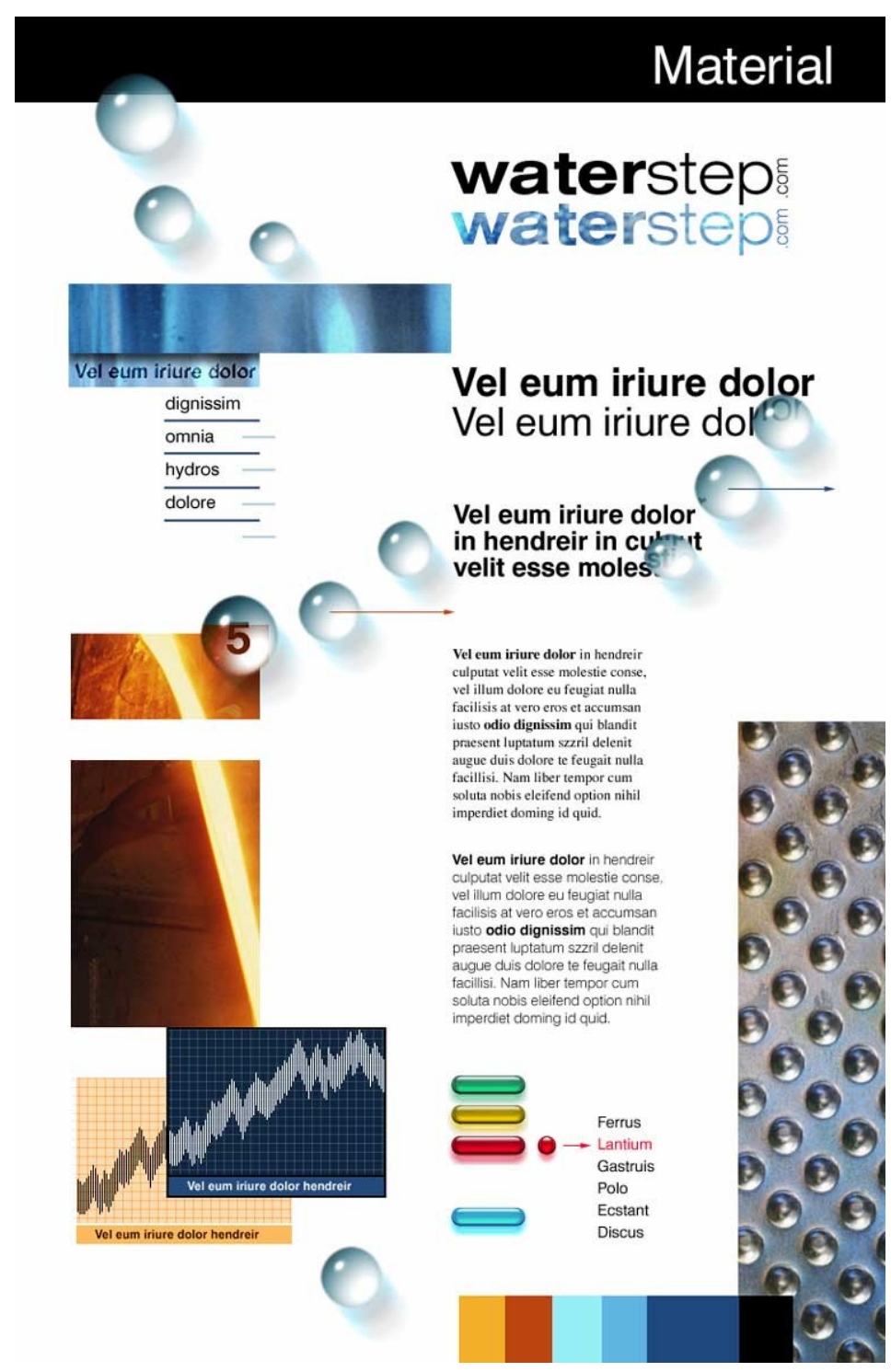

Figure 7. Exemple de concept board pour un prototype de site industriel (http://www.miguelmuelle.com/web/water.html)

Des sites organisés en feuille de style qui présentent le même contenu avec différentes ambiances graphiques pourront aider à la réflexion (CSS ZenGarden www.csszengarden.com). La combinaison du prototypage / zonage et de l'ambiance graphique pourra donner lieu au développement d'une charte fonctionnelle et/ou 
graphique. Dans cette étape qui consiste à organiser la partie visible du document on recourra massivement aux organisateurs para-linguistiques...

\section{Etape 3 Découpage à grain fin (DGF)}

\subsection{Procédure}

Le DGF consiste à considérer (approximativement) l'ensemble des informations qui sont présentées dans la zone principale des pages de contenu. Cette zone est généralement celle qui occupe le plus d'espace à l'écran. Selon les projets, cette zone doit encore être analysée pour un découpage homogène de son contenu. Quelle méthode choisir?

Tout d'abord, on peut distinguer plusieurs méthodes selon les types de texte présentés. Nous nous limiterons dans le cadre de cette réflexion aux textes techniques, utilitaires, qui sont les plus fréquemment rencontrés sur écran.

\subsection{Organiser selon une Macro-structure}

Une première question à se poser est : le texte à présenter sur écran fait-il partie de catégories de textes qui ont une organisation reconnue ? Certains textes ont en effet une « macro-structure » qu'il n'est pas possible ou souhaitable de modifier lors du passage à l'écran (textes juridiques, articles de psychologie expérimentale etc.) Dans ce cas, la segmentation de l'information va respecter les habitudes des usagers de ce type de textes, faute de quoi, le texte deviendra inutilisable pour les professionnels qui doivent s'en servir.

\subsection{Organiser selon l'importance et la nature des informations}

Concernant les autres types de textes techniques, en l'absence de macro-structure à respecter, on veillera à distinguer deux niveaux dans le texte. Ce qui est indispensable à la compréhension du texte ou à l'exécution d'une procédure décrite par le texte (informations de premier plan) et ce qui est accessoire (informations de second plan). Par analogie à une recette de cuisine, les unités textuelles de premier plan ce sont les ingrédients et la marche à suivre. Les informations de second plan sont toutes les autres informations de moindre importance (commentaires de l'auteur sur les trucs et astuces, sur la lecture du texte, également l'identification de l'auteur, le niveau d'expérience du public visé, le niveau de difficulté de la recette, les vins qui peuvent accompagner le plat, etc.) La figure 8 présente un exemple de distinction premier plan / second plan par la mise en forme matérielle du texte. 


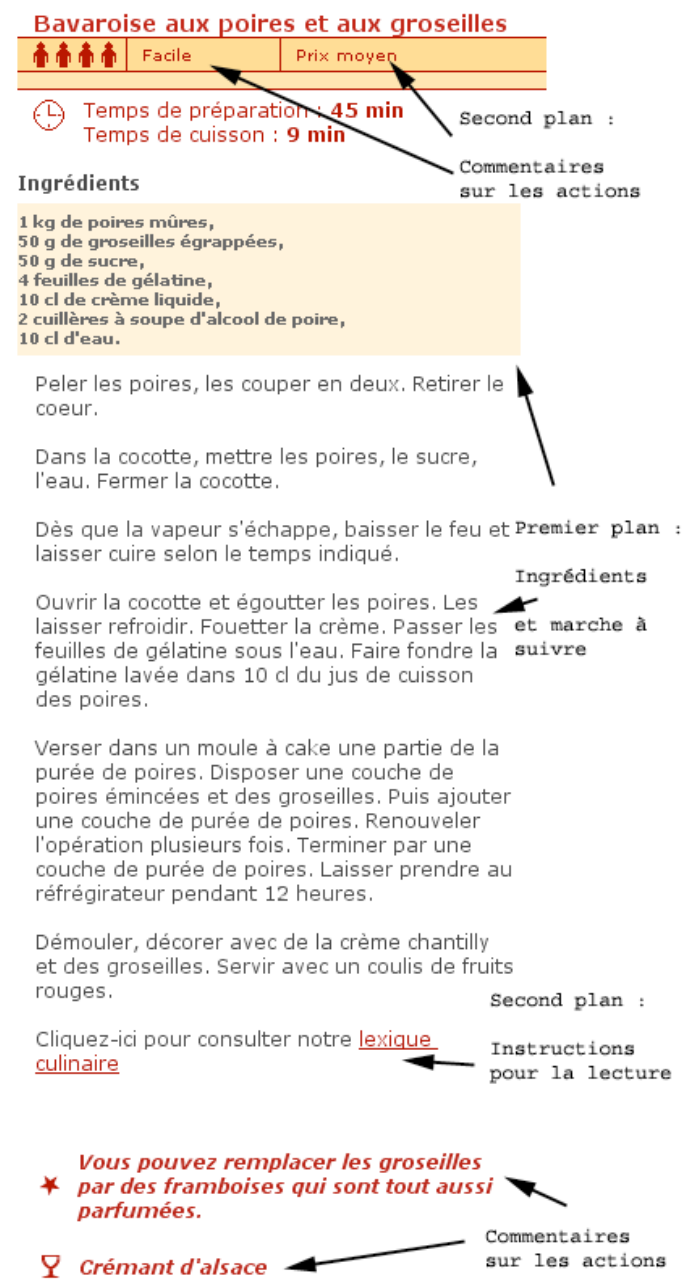

Figure 8. Illustration de la distinction premier plan second plan Site Internet www.tefal.fr

\subsection{Organiser pour faciliter la sélection d'informations}

Enfin dans de nombreux autres cas et notamment pour les écrits de type journalistiques, la méthode de pyramide inversée convient bien à la lecture à l'écran. Elle consiste à donner les informations les plus importantes en premier (titre et chapeau) et à donner plus de précision au lecteur qui continuera la lecture du texte. Le chapeau, petit résumé du texte, placé au début de celui-ci permettra en général au lecteur de lire (ou de se dispenser) du reste à bon escient. La méthode des cinq W, 
(Who) qui, (What) quoi, (When) quand, (Where) où, (Why) pourquoi, également issue de la presse, convient aussi pour les textes sur écran (Bailly, 2003). Elle permet de s'assurer que toutes les informations nécessaires à la construction d'une représentation de ce que dit le texte sont présentées au lecteur. Cette construction est pertinente pour des textes dont la lecture est fragmentaire (lecture de consultation plutôt que « de bout en bout »).

\subsection{Mise en relief de l'information}

Une fois le texte découpé et organisé de manière homogène et cohérente, les différentes parties seront signalées par des organisateurs de mise en forme ou «paralinguistiques ». Ces organisateurs pourront permettre de distinguer les informations de premier plan et de second plan mais aussi les différents types d'informations au sein de chaque niveau d'importance (ingrédients, marche à suivre, commentaires sur les actions, sur le niveau d'expérience du lecteur etc.) On veillera à rester homogène quant aux organisateurs choisis pour la mise en forme de chaque type d'information.

\section{Les organisateurs para-linguistiques}

Les organisateurs para-linguistiques sont liés à la mise en relief de l'information par des moyens non-langagiers. Une manière langagière de marquer une information de moindre importance peut consister à faire précéder celle-ci d'un marqueur textuel «...soit dit entre parenthèses, [...] ». Dans l'espace limité de l'écran, la mise en relief se fait souvent par le moyen d'organisateurs graphiques plutôt que langagiers.

Leur fonction est double :

- indiquer l'importance d'une information (mise en avant ou mise en retrait),

- indiquer la nature des informations (commentaire de l'auteur, exemple, définition, référence...)

On distingue habituellement les organisateurs para-linguistiques suivant :

- l'enrichissement typographique (variations de polices de caractère),

- les encadrés, aplats colorés, filets et autres dispositifs de présentation synoptiques (organigrammes tableaux),

- la couleur,

- l'espace et la densité informative,

- la ponctuation,

- mise en note, parenthèses,

- les caractères spéciaux, icônes. 
mais aussi ceux qui sont plus spécifiques des documents numériques :

- les animations d'objet ou de texte (clignotement, défilement, inverse vidéo, effets de flash),

- le temps (affichage temporisé de parties du texte par exemple),

- les escamots (pop-up window),

- le multifenêtrage,

- la transparence,

- les effets de flou,

- les effets fish eye ou loupe.

\section{Conclusion}

Bien entendu lors des différentes étapes et pour tous les livrables, le commanditaire doit valider les choix de l'agence (cahier des charges, cahier de conception avec l'arborescence ou le scénario, les maquettes des écrans type, le découpage de l'information dans les zones d'information principale, la charte graphique / fonctionnelle...). Même si ces étapes reviennent fréquemment dans les projets de conception de documents numériques, la procédure peut revêtir également une part de nouveauté selon les projets, auquel cas l'activité des concepteurs est planifiée de façon opportuniste. Dans ce cas l'ordre habituel des étapes pourra être quelque peu bousculé (le concepteur commence par ce qu'il connait sans nécessairement procéder du plus global au spécifique) et intervient à différents niveaux de la conception (structure, détails) (Cf. la préface d'André Bisseret de l'ouvrage (Caro, 2007)). En dépit de ces situations de conception assez fréquentes, il nous a semblé nécessaire de donner un aperçu d'un parcours de conception que l'on pourrait qualifier de «standard ». Nous n'avons toutefois pas présenté la totalité des méthodes qui peuvent être mobilisées, préférant comme annoncé dans cette introduction, donner un développement plus précis à quelques unes d'entre elles. 


\section{Bibliographie}

Bailly S. Bien écrire pour le Web. Paris, Osman Eyrolles Multimédia, 2003.

Bisseret A., Sebillotte S., Falzon P., Techniques pratiques pour l'étude des activités expertes, Toulouse, éditions Octarès, Collection Travail dirigée par François Daniellou et Gilbert de Terssac, 1999,.

Caro S. L'écriture des documents numériques : approche ergonomique. Paris, Hermès Editeur, collection ingenierie représentationnelle et construction de sens dirigée par Sylvie LeleuMerviel, 2007.

Ericsson K.-A., Simon H., Protocol analysis : verbal reports as data, (revised edition), Cambridge, MA. MIT Press, 1993.

Kintsh W., van Dijk T.-A., «Vers un modèle de la compréhension et de la production de textes », in Il était une fois Compréhension et souvenir de récits. Presses Universitaire de Lille. Textes traduits et présentés par Guy Denhière, Lille, 1978, pp. 85-142.

Kintsh W., "The role of knowledge in discourse comprehension : A constructionintegration », Psychological Review, n ${ }^{\circ}$ 95, 1988, pp. 163-182.

Kintsh W., Comprehension a paradigm : for cognition, New York : Cambridge, University Press, 1998.

Poulson D., Ashby M., Richardson S., USERfit, A practical handbook on user-centred design for Assistive Technology, ESC-EC-EAEC, Brussels-Luxembourg, Editors: David Poulson, Martin Ashby, Simon Richardson, HUSAT Research Institute, UK, 1996.

Ronez J. L'écrit Web : Traitement de l'information sur Internet. Paris, CFPJ Editions, 2007.

Scapin D.-L., Pierret-Golbreich C., «Towards a method for task description », actes de la conférence Working with display units, The Netherlands L. Berlinguet, D. Berthelette (editors), Amsterdam, Elsevier, 1990, pp. 371-380. 